\section{A novel stent for management of duodenal obstruction}

A 61-year-old woman was admitted for acute pancreatitis after endoscopic retrograde cholangiopancreatography (ERCP). At 1 week after standard treatments, the symptoms of pancreatitis disappeared. However, the patient could not eat anything, vomited repeatedly and developed recurrent biliary infections. Computed tomography $(\mathrm{CT})$ revealed an enlarged pancreatic head $(4 \times 6 \mathrm{~cm}$; $\odot$ Fig. 1 a). A jejunal tube and a nasogastric tube were introduced. The drainage volume was around $1500 \mathrm{ml} /$ day and did not decrease in the following 2 months. Iodine swallow revealed an obstruction at the distal descending duodenum and the proximal horizontal duodenum ( $\bullet$ Fig. 1 b).

Postpancreatitis duodenal obstruction is rare, the incidence being as low as $1.2 \%$ [1], and it is mostly mild to moderate. There is a high mortality rate among patients with severe events such as that described here. Surgery is generally indicated when the obstruction persists for more than 4 weeks. However this patient had already previously undergone a choledocholithotomy and a cholecystectomy. The difficulty and risk associated with a third surgical procedure was extremely high.

We propose that temporary metallic stenting is a feasible method for managing postpancreatitis duodenal obstruction. We therefore designed a retrievable metallic stent with a drawstring. The stent is round-tipped, machine-woven, and noncoated, with a metal retrieval line that extends out of the body. Because the retrieval line is thin, all procedures were performed under digital subtraction angiography (DSA).

After preparation had been completed, the stent was successfully placed at the site of obstruction site ( $\bullet$ Fig. 1 c); the retrieval line extended extracorporeally
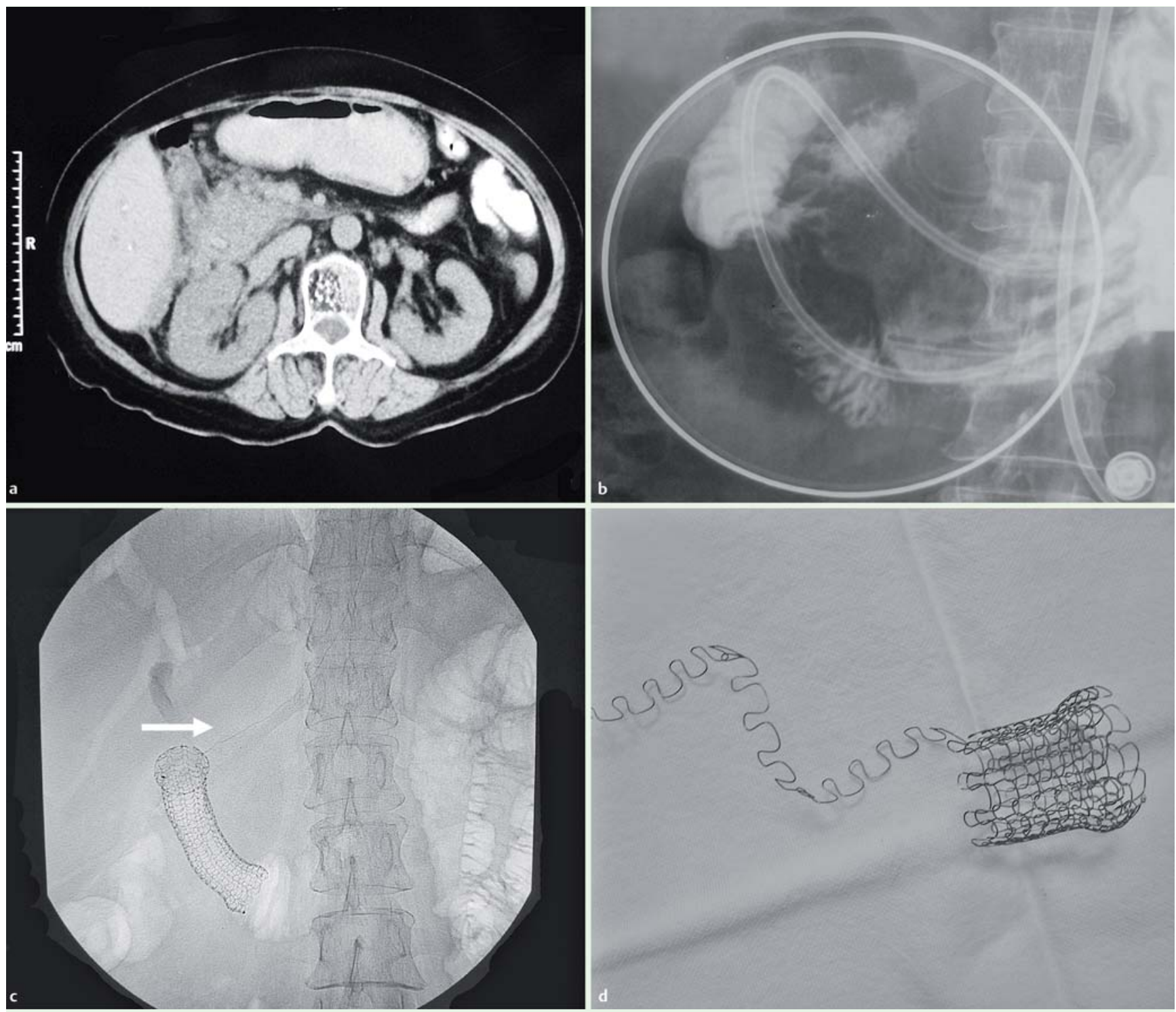

Fig. 1 a Computed tomography (CT) revealed an enlarged pancreatic head. b lodine swallow demonstrated an obstruction at the distal descending duodenum and the proximal horizontal duodenum. $\mathbf{c}$ The stent was positioned at the obstruction site (arrow: the retrieval line). $\mathbf{d}$ The novel stent. 
from the proximal tip. On the same day as the procedure, the patient was able to eat semifluid foods. The stent was removed 1 week later, being unravelled ( $\bullet$ Fig. 1 d) by drawing the retrieval line. There was no evidence of recurrence 2 years postoperatively.

We believe that this novel stent also provides an effective method for managing benign strictures in other parts of the gastrointestinal tract.

Endoscopy_UCTN_Code_TTT_1AO_2AZ
H. Cai ${ }^{1}$, X. Jiang ${ }^{2}$, Q. Dong ${ }^{1}$, H. Li ${ }^{1}$, W. $\mathrm{Xie}^{1}$

1 Department of Gastroenterology, Changzheng Hospital, the Second Military Medical University, Shanghai, China

2 Department of Anesthesiology, Changzheng Hospital, the Second Military Medical University, Shanghai, China

\section{References}

1 Vijungco JD, Prinz RA. Management of biliary and duodenal complications of chronic pancreatitis. World J Surg 2003; 27: 1258 1270
Bibliography

DOI $10.1055 / \mathrm{s}-0029-1214436$

Endoscopy 2009; 41: E254-E255

(c) Georg Thieme Verlag KG Stuttgart · New York . ISSN 0013-726X

\section{Corresponding author}

W. Xie, MD, PhD

Department of Gastroenterology

Changzheng Hospital

The Second Military Medical University

Shanghai

China

Fax: +86-21-63610109

czxieweifen@163.com 